\title{
Correção atmosférica e de "crosstalk" em dados Aster para mapeamento da relação mineralógica de solos
}

\author{
Gustavo Macedo de Mello Baptista(1), Daniella Dias Vivaldi(1) e Paulo Roberto Meneses ${ }^{(1)}$
}

(1)Universidade do Brasília, Instituto de Geociências, Campus Darcy Ribeiro, Asa Norte, Caixa Postal 4.465, CEP 70910-900 Brasília, DF, Brasil. E-mail: gmbaptista@unb.br, dvivaldi@gmail.com, pmeneses@unb.br

\begin{abstract}
Resumo - O objetivo deste trabalho foi avaliar a influência das correções atmosférica e de "crosstalk" (vazamento de radiação entre detectores) da qualidade das imagens RCGb $\mathrm{Bcale}_{\text {,e }}$ em dados SWIR do Aster, sobre a identificação da relação mineralógica RCGb [caulinita/(caulinita + gibbsita)], em uma mancha de solos expostos derivados do Complexo de Niquelândia, composto por gabronoritos, noritos e piroxenitos. Utilizaram-se dados brutos L1A, L1B (radiância no topo da atmosfera), produtos AST07 (reflectância de superfície sem correção de "crosstalk") e AST07XT (reflectância de superfície com correção de "crosstalk"), os módulos de correção atmosférica Flaash e Quac, e o programa de correção "crosstalk" CRSTK30. Os resultados foram ordenados de acordo com os seguintes parâmetros: os transectos $\mathrm{RCGb}_{\text {scale }}$ e $\mathrm{RCGb}_{\mathrm{ATG}}$; a identificação da espacialização da relação mineralógica nas imagens $\mathrm{RCGb}_{\text {scale, }}$, fatiadas em classes de valor Ki; e a verificação quantitativa do grau de similaridade entre os valores dos 17 pontos dos transectos $\mathrm{RCGb}_{\text {scale }}$ e $\mathrm{RCGb}_{\mathrm{ATG}}$, por meio dos testes estatísticos t de Student e U de Mann-Whitney. Os procedimentos apresentaram 75\% de resultados satisfatórios para o estudo proposto. Os dados L1B, bem como os efeitos atmosféricos e os do "crosstalk", não interferem na identificação da relação RCGb. Estudos em outros solos devem ser incentivados para melhor compreensão da interferência dessas correções nos dados Aster.
\end{abstract}

Termos para indexação: Complexo de Niquelândia, minerais opacos, relação mineralógica RCGb, sensoriamento remoto.

\section{Atmospheric and crosstalk corrections in Aster data for the mapping of soil mineralogical relationship}

\begin{abstract}
The objective of this work was to evaluate the effect of atmospheric and crosstalk (the electrical or optical signals of band leaks into another band) corrections of $\mathrm{RCGb}_{\text {scale }}$ image quality, in Aster SWIR data, on the identification of the mineralogical relationship RCGb [kaolinite/(kaolinite + gibbsite)], in a spot of bare soils derived from the Niquelândia Complex, composed by gabbronorites, norites and pyroxenites. Raw data were used for L1A, L1B (radiance at the top of the atmosphere), AST07 (surface reflectance without crosstalk correction) and AST07XT (surface reflectance with crosstalk correction) on-demand products, the atmospheric correction modules Flaash and Quac, and the CRSTK30 crosstalk correction program. The results were sorted according to the following parameters: the transects $\mathrm{RCGb}_{\text {scale }}$ and $\mathrm{RCGb}_{\mathrm{ATG}}$; the identification of the spatialization of the mineralogical relationship in the $\mathrm{RCGb}_{\text {scale }}$ images, which were sliced into Ki value classes; and the quantitative check of the similarity degree between the values of 17 points of the $\mathrm{RCGb}_{\text {scale }}$ and $\mathrm{RCGb}_{\mathrm{ATG}}$ transects, by Student's $\mathrm{t}$ and Mann-Whitney U statistical tests. The procedures showed $75 \%$ satisfactory results for the proposed study. The L1B data, as well as the atmospheric and crosstalk effects, do not interfere in the identification of the RCGb relationship. Studies in other soils should be stimulated for a better understanding of the interference of these corrections in Aster data.
\end{abstract}

Index terms: Niquelândia Complex, opaque minerals, RCGb mineralogical relationship, remote sensing.

\section{Introdução}

Em solos que apresentam elevados estágios de intemperismo, observa-se a remoção de sílica e bases trocáveis e o posterior acúmulo de alumínio. Os Latossolos apresentam teores variáveis de caulinita e gibbsita em sua fração argila, além de outros minerais como os sesquióxidos de ferro. A caulinita tem em sua composição química o percentual restante da sílica, e a gibbsita apresenta em sua composição tanto a sílica quanto a alumina. Por isso, a relação mineralógica 
RCGb: caulinita/(caulinita+gibbsita) pode indicar o valor $\mathrm{Ki}$ dos solos tropicais, que apresenta a relação molecular $\left[\mathrm{SiO}_{2}\right] /\left[\mathrm{Al}_{2} \mathrm{O}_{3}\right]$ (Madeira Netto, 1993; Baptista, 2001).

A relação entre esses dois minerais possibilita entender o grau de intemperismo das coberturas pedológicas dos Latossolos, o que pode contribuir para o entendimento da dinâmica físico-química que influencia a produtividade e, consequentemente, a nutrição de plantas, e pode, também, intervir em fenômenos relacionados ao manejo e à conservação do solo, em caso de uso deste para plantio (Baptista et al., 2011).

As práticas usuais de investigação mineralógica pressupõem a coleta de amostras de solos em campo, em pontos aleatórios ou com uso de grades regulares, com o objetivo de compreender a variabilidade espacial e, assim, direcionar a aplicação de insumos para suprir as demandas nutricionais das culturas. Porém, às vezes, essas técnicas tradicionais não conseguem investigar de forma acurada as variações disponíveis no campo sendo muito dispendiosas, pois, requerem um grande número de amostras (Baptista et al., 2011).

Para minimizar os custos e aumentar a eficiência da aplicação de insumos, bem como a produtividade, essas investigações mineralógicas são cada vez mais possíveis por meio de técnicas de sensoriamento remoto que permitem a identificação dos minerais, geralmente, por meio do método de comparações entre os espectros de reflectância dos alvos da imagem e os espectros de referência dos minerais das amostras de campo - ou de bibliotecas espectrais disponíveis nos próprios programas ou literaturas inerentes à investigação de minerais (Madeira Netto, 1993).

Entre os diversos sistemas sensores colocados em órbita nos últimos anos, o Aster ("Advanced Spaceborne Thermal Emission and Reflection Radiometer") foi concebido para ter a possibilidade de separação dos principais minerais da fração argila associados às hidroxilas, que apresentam feições espectrais centradas em 2,0 $\mu \mathrm{m}$, bem como dos carbonatos, entorno de 2,3 $\mu \mathrm{m}$ (Galvão et al., 2005; Abrams et al., 2007). Como a caulinita apresenta sua principal feição em 2,205 $\mu \mathrm{m}$, e a gibbsita, em $2,265 \mu \mathrm{m}$, essas duas feições estariam contempladas paras as manchas de solos tropicais expostos, principalmente os formados por latolização (Madeira Netto, 1993).
A quantificação dessa relação mineralógica em dados Aster foi possível por meio do desenvolvimento do índice $\mathrm{RCGb}_{\text {scale }}$ (Baptista, 2001) - originalmente pensado para dados hiperespectrais -, obtido a partir das imagens scale, que são produtos resultantes da aplicação do algoritmo "spectral feature fitting" (Clark et al., 1990) que representa a intensidade das feições espectrais que, segundo Baptista (2001), são proporcionais ao conteúdo do endmember, também conhecido como espectro de referência investigado.

Ao testar o índice $\mathrm{RCGb}_{\text {scale, }}$ em dados do sensor SWIR do sistema Aster, Baptista et al. (2007) perceberam o deslocamento das feições de absorção dos minerais caulinita e gibbsita, bem como a obliteração do ponto de máxima reflexão existente entre as duas principais feições, típicas dos espectros de reflectância de solos que apresentam esses minerais.

Baptista etal. (2007) relacionaram esse deslocamento ao efeito "crosstalk", entendido como um "vazamento" de radiação dos detectores da banda 4 para os detectores das bandas vizinhas 5 e 9, que levou ao comprometimento dos dados e ao descomissionamento do sensor SWIR a partir de abril de 2008 (Vivaldi et al., 2013). Caberia uma investigação de quanto esses efeitos poderiam permitir a quantificação e espacialização da relação mineralógica.

O objetivo deste trabalho foi avaliar a influência das correções atmosférica e de "crosstalk" (vazamento de radiação entre detectores) da qualidade das imagens RCGb $b_{\text {scale, }}$ em dados SWIR do Aster, sobre a identificação da relação mineralógica RCGb [caulinita/ (caulinita + gibbsita)], em uma mancha de solos expostos derivados do Complexo de Niquelândia, composto por gabronoritos, noritos e piroxenitos.

\section{Material e Métodos}

A área de estudos é uma mancha de solos localizada na Zona Máfica Inferior (ZMI), na porção leste do Complexo de Niquelândia, e é constituída principalmente por gabronoritos, noritos e piroxenitos (Ferreira Filho et al., 2010). A escolha dessa área de estudos se justifica pelo fato de os solos da área apresentarem um limitador aos estudos espectrais de solos tropicais, em razão da presença de magnetita, mineral opaco que tende a obliterar as feições espectrais dos minerais da fração argila de solos tropicais.

Pesq. agropec. bras., Brasília, v.51, n.9, p.1272-1282, set. 2016 DOI: 10.1590/S0100-204X2016000900027 
Ferreira Filho et al. (2010) consideram que o Complexo de Niquelândia, com cerca de $1.800 \mathrm{~km}^{2}$ de área, e espessura estimada de 10 a $15 \mathrm{~km}$, e com litologias bem expostas como o formado por duas intrusões distintas e não relacionadas, ou seja, é composto por duas sequências superpostas, petrologicamente distintas e tectonicamente justapostas.

A primeira sequência apresenta a série acamadada inferior (SAI), a leste (E), e é constituída por três zonas de rochas acamadadas, com imersão para oeste (W), que seguem o sentido: Zona Máfica Inferior (ZMI), Zona Ultramáfica (ZU) e Zona Máfica Superior (ZMS). A segunda sequência apresenta a série acamadada superior (SAS), a oeste (W), conhecida anteriormente como Unidade Serra dos Borges. A Figura 1 apresenta o esboço geológico do Complexo de Niquelândia realizado por Ferreira Filho et al. (2010).

A ZMI - local da mancha de solos investigada no presente estudo - é constituída por gabronorito com websterito e norito interacamadados. Essa zona é descrita por Ferreira Filho et al. (2010) como uma zona de acumulação basal, localizada estratigraficamente abaixo da ZU. A ZU é constituída por sequências de unidades cíclicas com dunito, harzburgito, websterito e ortopiroxenito interacamadados e lherzolito e cromitito subordinados. O contato superior da ZU é gradacional para a ZMS e caracterizado pela diminuição gradativa da frequência de camadas de rochas ultramáficas (Ferreira Filho et al., 2010).

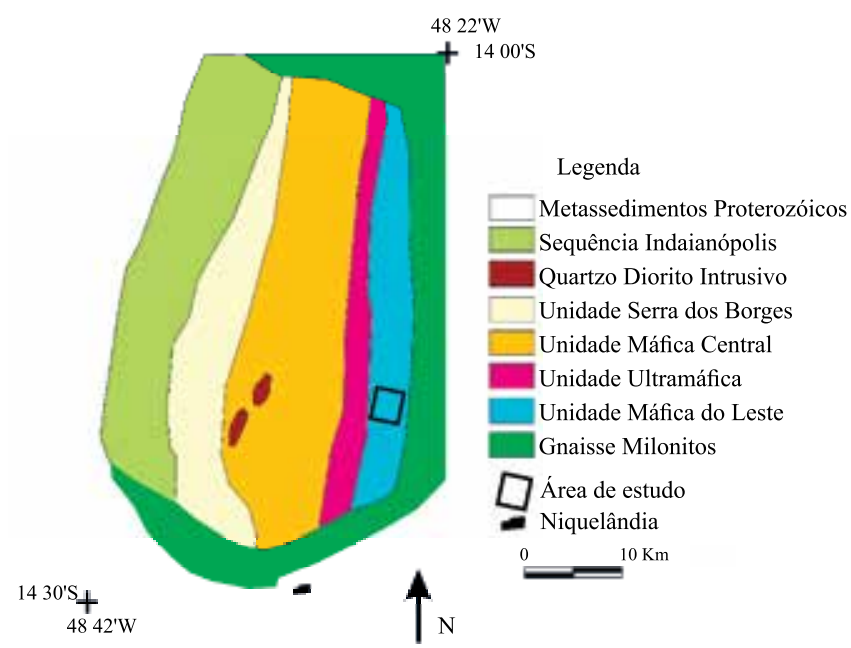

Figura 1. Esboço geológico do Complexo de Niquelândia (GO), com destaque da área de estudos. Fonte: adaptado de Ferreira Filho et al., (2010).
Os últimos estudos geocronológicos relativos à área indicam que a SAS tem idade mesoproterozoica (cerca de $1,25 \mathrm{Ga}$ ) e é, portanto, mais velha que a SAI, que tem, provavelmente, idade neoproterozoica de cerca de 0,79 Ga (Ferreira Filho et al., 2010).

Nas formações superficiais ou coberturas pedológicas na mancha investigada, verificou-se, em campo, um solo do tipo Argissolo Vermelho, no contato com a Zona Ultramáfica, e uma gradação para um Latossolo Vermelho distroférrico numa topossequência de 1.700 $\mathrm{m}$, avaliada com espaçamento de $100 \mathrm{~m}$ entre os pontos, na porção NE da área de estudo. Os Argissolos ocorrem em leques aluviais provenientes do topo do maciço (ZU), associadas à zona de acúmulos basais, enquanto os Latossolos ocorrem em porções mais erodidas da área de estudo, conforme salienta Baptista et al. (2011), ao mapear essa mesma área com dados Aviris. Esses solos derivados das rochas do complexo, normalmente, apresentam em sua composição minerais opacos, em especial a magnetita.

O Município de Niquelândia está situado na macrounidade geomorfológica de planaltos, em cinturões orogênicos de planaltos e serras de Goiás-Minas, que são divisores de águas das bacias hidrográficas de Tocantins e São Francisco. Essa macrounidade é caracterizada como topo de vertente convexa, declinada a $3 \%$ no sentido NW-SE. A topografia varia de plano a suavemente inclinado (Baptista, 2001). Tem superfície aplainada intermediária, bordeada por estrutura mais soerguida. Apresenta superfície de gradiente suave, que varia entre 3 e $8 \%$ de declividade, o que favorece o intemperismo químico a profundidades significativas por terem alto grau de permeabilidade vertical (Baptista, 2001).

Essa área de estudos encontra-se sob a influência do clima tropical, do tipo Aw, segundo a classificação de Köppen-Geiger, com concentração da precipitação pluvial no período do verão. Os meses mais chuvosos são novembro, dezembro e janeiro, e a época seca ocorre nos meses de inverno, ou seja, de junho a agosto (Baptista, 2001).

O Aster é um dos cinco instrumentos de imageamento terrestre a bordo do satélite Terra e realiza a obtenção das informações da superfície terrestre por meio de 14 bandas espectrais distintas, distribuídas em três subsistemas: o "Visible and Near Infrared Radiometer" (VNIR); o "Short-Wave Infrared" (SWIR); e o "Thermal Infrared" (TIR) (Abrams et al., 2007). 
Após o lançamento do satélite Terra, observou-se no subsistema SWIR um "vazamento" de radiação dos detectores da banda 4 para os detectores das bandas vizinhas 5 e 9 . O motivo para que esse "vazamento" foi identificado apenas na banda 4 e pode estar relacionado às características desta que é a banda de maior amplitude e maior potência de refletividade do SWIR, com registro de radiação cerca de quatro a cinco vezes mais intensa do que as demais bandas. Esse vazamento de sinal foi nomeado como "crosstalk", o qual ainda é agravado pelo efeito do erro de paralaxe e pela distância entre os pares de matriz CCD (Iwasaki $\&$ Tonooka, 2005). Esse efeito é destacado na Figura 2, na qual se pode verificar o espectro do mesmo pixel de solo exposto, pixel 13 do transecto aqui estudado, que mostra a presença de caulinita e de gibbsita numa imagem Aviris reamostrada para o Aster, sem o “crosstalk", e para o pixel Aster que apresenta o efeito deslocando o espectro nas bandas 5 e 9 .

O erro de paralaxe do SWIR é consequência do deslocamento no alinhamento do detector (offset), na direção do deslocamento da plataforma, em função da distância entre a plataforma e a superfície observada na Terra. Para a correção desse tipo de erro, geralmente, utilizam-se técnicas de combinação (matching) ou de dados de modelos numéricos de terreno (Iwasaki \& Tonooka, 2005).

Os produtos Aster selecionados para o presente estudo e suas especificações são apresentados na Tabela 1.

O Aster produz dois tipos de dados de nível 1: L1A e L1B. Os produtos L1A são formados por dados digitais (DN), reconstruídos dos três subsistemas, ou seja, são os dados originais da radiância capturada pelos subsistemas, sem qualquer calibração dos coeficientes, seja ela radiométrica, geométrica ou de erro de paralaxe, assim como qualquer tipo de correção para efeitos de fenômenos naturais ou correções do próprio subsistema. Os produtos $\mathrm{L} 1 \mathrm{~B}$, radiância no topo da atmosfera ("top of the atmosphere" - TOA), os coeficientes das calibrações geométricas, radiométricas e de paralaxe já se encontram aplicados (Iwasaki \& Tonooka, 2005).

Os produtos AST07 e AST07XT, obtidos sob demanda, apresentam a aplicação dos coeficientes das calibrações e da correção atmosférica. No caso do AST07XT, a correção do efeito "crosstalk" também se encontra aplicada por meio de algoritmo próprio (Mars \& Rowan, 2010).

Ponto 13

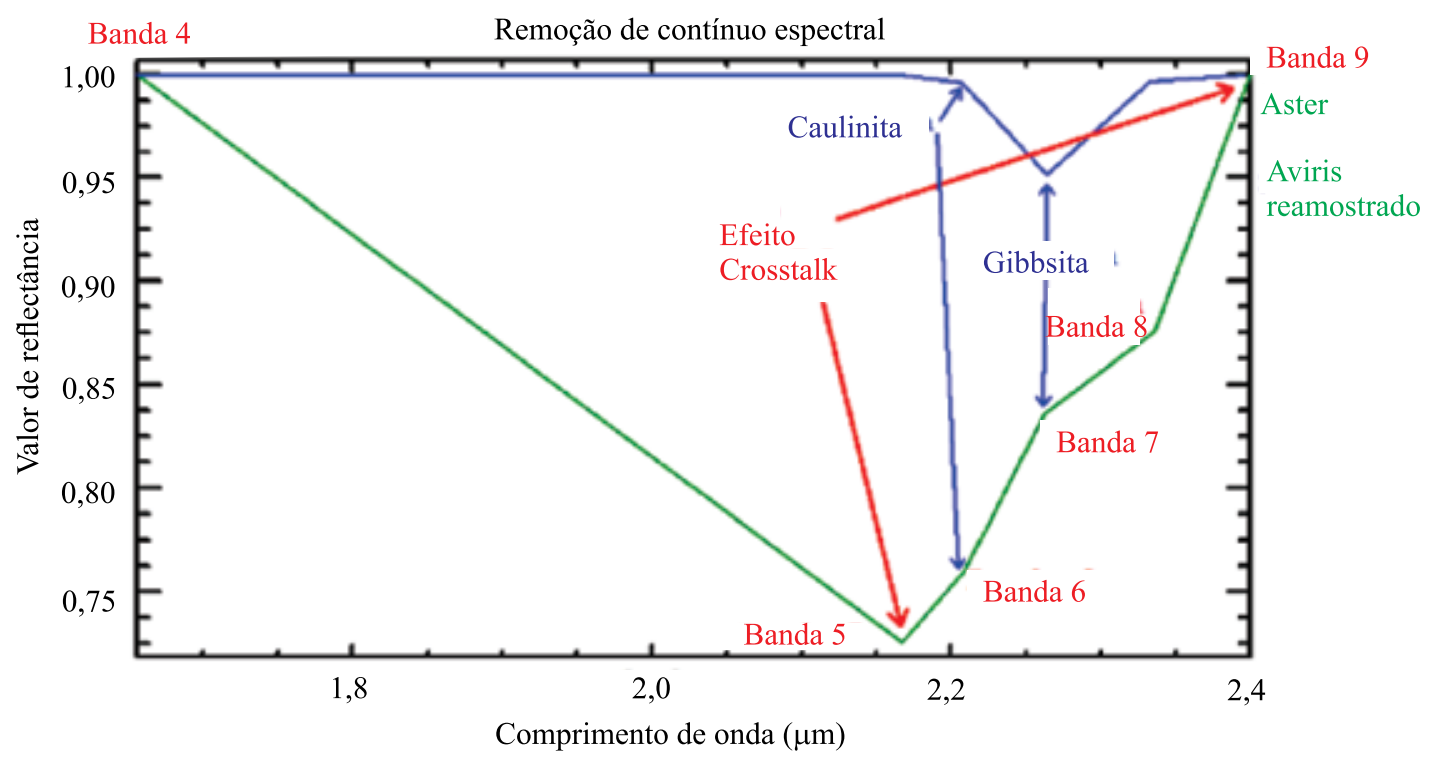

Figura 2. Efeito crosstalk em dados Aster, observado para o mesmo pixel de solo exposto obtido sem esse efeito por dados Aviris e reamostrados para Aster. 
Os procedimentos metodológicos foram divididos em duas etapas: pré-processamento e processamento dos dados, que foram realizados por meio do programa "Environment for Visualizing Images" (Envi), que foi escolhido por apresentar o conjunto de aplicativos mais completo e adequado para a execução do presente estudo.

A etapa de pré-processamento foi estabelecida para padronizar os dados para uma melhor análise e comparação. Primeiramente, realizou-se a reamostragem espacial de 30 para $15 \mathrm{~m}$, por meio do módulo "resize data". Esse processamento foi empregado tanto nos dados de radiância (L1A e L1B) como nos de reflectância, obtidos sob demanda (AST07 e AST07XT) para agrupar os dados dos sensores VNIR e SWIR, que possuem resoluções espaciais diferentes, e esse procedimento é necessário para a aplicação dos algoritmos de correção atmosférica e de "crosstalk".

Os dados L1B foram convertidos para dados de reflectância no TOA ou, sem a correção, para os efeitos atmosféricos, por meio do módulo "flat field", que consiste num método simples de normalização dos dados de radiância para reflectância aparente (TOA), baseado numa área espectral plana sem grandes variações, ou seja, a mais homogênea possível ou a mais uniforme espectralmente (Vivaldi et al., 2013).

Para a correção atmosférica, utilizaram-se os módulos dos algoritmos "fast line-of-sight atmospheric analysis of spectral hypercubes" (Flaash) e "quick atmospheric correction" (Quac) em dados L1A (dados brutos de radiância TOA). O Flaash utiliza o código de transferência radiativa MODTRAN4, executa a correção para o efeito de adjacência (mistura de pixel em razão do espalhamento de superfície) e, também, calcula a visibilidade média da cena (aerossol/névoa) (Perkins et al., 2012). O Quac possui velocidade computacional significativamente mais rápida, efetua a correção atmosférica a partir de modelos empíricos, com uso somente das informações contidas na cena, ou seja, o desvio-padrão espectral do conjunto de materiais diversos existentes na cena (Bernstein et al., 2012).

Para a correção do efeito do "crosstalk", o programa utilizado foi o CRSTK30, desenvolvido pela ERSDAC ("Earth Remote Sensing Data Analysis Center"), para ser aplicado sobre os dados de entrada do produto L1B. Segundo Iwasaki \& Tonooka (2005), o efeito está presente tanto nos dados L1A como no L1B, e apenas o fluxo de processamento mudaria, porém, esses autores destacam que o efeito é bem mais sentido nos dados L1B, daí a opção de entrada no programa desses dados. A aplicação do índice $\mathrm{RCGb}_{\text {scale, }}$ que caracteriza a etapa de processamento, pressupõe que as cenas tenham sido normalizadas por meio da remoção do contínuo espectral, e que seja aplicado o algoritmo "spectral feature fitting" (SFF), que é um ajuste por mínimos quadrados entre o espectro do pixel da cena e o espectro do "endmember" (referência). Ele gera dois resultados: as imagens scales, que representam a intensidade ou a profundidade da feição espectral; e as imagens RMS, que representam o erro médio do ajuste por mínimos quadrados. O RCGb $\mathrm{Rcale}_{\text {é }}$ baseado nas imagens scale.

Em seguida, realizou-se a reamostragem dos "endmembers" a serem investigados na cena, por meio dos espectros dos minerais "kaolinite well ordered" OS-1A e "gibbsite synthetic" OH-3A da biblioteca espectral do JPL, para as funções de resposta espectral dos detectores do Aster (Baldridge et al., 2009). Esses "endmembers" foram escolhidos por serem das mesmas amostras utilizadas por Baptista (2001), quando do desenvolvimento do algoritmo $\mathrm{RCGb}_{\text {scale. }}$.

A aplicação do índice $\mathrm{RCGb}_{\text {scale, }}$ principal passo dessa etapa, foi realizada por meio da expressão $b_{1} /\left(b_{1}+b_{2}\right)$, inserida no módulo "band math", em que: $b_{1}$ refere-se à imagem scale kaolinite, e $b_{2}$ à scale gibbsite.

$\mathrm{O}$ índice $\mathrm{RCGb}$ scale foi aplicado sobre os dados, que passaram pelas seguintes etapas de pré-processamento: L1A (dados brutos), sem as correções atmosférica e de

Tabela 1. Produtos Aster utilizados no estudo.

\begin{tabular}{|c|c|c|c|c|c|c|c|}
\hline \multirow[t]{2}{*}{ Nome } & \multirow[t]{2}{*}{ Produto } & \multirow{2}{*}{$\begin{array}{c}\text { Resolução } \\
\text { espacial (m) }\end{array}$} & \multicolumn{3}{|c|}{ Calibração - coeficiente de correção } & \multicolumn{2}{|c|}{ Correção } \\
\hline & & & Geométrica & Radiométrica & Paralaxe & Atmosférica & Crosstalk \\
\hline ASTL1A & Radiância (dados brutos) & 30 & Não & Não & Não & Não & Não \\
\hline ASTL1B & Radiância registrada no sensor & 30 & Sim & Sim & Sim & Não & Não \\
\hline AST07 & Reflectância & 30 & $\operatorname{Sim}$ & $\operatorname{Sim}$ & $\operatorname{Sim}$ & $\operatorname{Sim}$ & Não \\
\hline AST07XT & Reflectância & 30 & Sim & Sim & Sim & Sim & Sim \\
\hline
\end{tabular}


"crosstalk"; L1B (radiância registrada no sensor), sem correções atmosférica e de "crosstalk"; L1B convertidos para reflectância TOA (flat field), sem correções atmosférica e de "crosstalk"; L1B convertidos para reflectância TOA (flat field), sem correção atmosférica e com correção do "crosstalk"; L1A convertida para reflectância de superfície (Flaash), sem correção do "crosstalk"; L1A convertida para reflectância de superfície (Quac), sem correção do "crosstalk"; L1B convertida para reflectância de superfície (Flaash), com correção "crosstalk"; L1B convertida para reflectância de superfície (Quac), com correção "crosstalk"; AST07 de reflectância de superfície, sem correção "crosstalk"; AST07XT de reflectância de superfície, com correção "crosstalk".

Com a finalidade de elucidar questões quanto à comparação entre os dados da imagem de radiância e as feições espectrais dos minerais, o presente estudo baseou-se nas seguintes premissas: considerou que as feições espectrais dos minerais, que são eventos quânticos, estão nos dados de radiância e não nos de irradiância; e, considerou que tanto os dados da imagem de radiância quanto as feições de espectros foram normalizados, por meio da remoção do contínuo espectral, quando se aplicou o módulo de classificação SFF.

Assim, após a normalização dos dois tipos de dados, a sua variação passa a ser a mesma, expressa em percentual e não mais em $\mathrm{W} \mathrm{m}^{-2} \mathrm{sr} \mu \mathrm{m}$. Se não houvesse a etapa de normalização dos dados, as unidades se manteriam diferentes, o que inviabilizaria a comparação.

Sobre cada imagem $\mathrm{RCGb}_{\text {scale }}$ obtida, foi traçado o transecto relativo a $1.700 \mathrm{~m}$, tendo-se obedecido o sentido NE-SO, do qual foram selecionados 17 pontos, com espaçamento de $100 \mathrm{~m}$ entre eles. Os conteúdos de caulinita e gibbsita foram obtidos conforme descrito por Plante et al. (2009), por meio de análise termogravimétrica (ATG) das amostras de solo coletadas em campo (Baptista, 2001). Em seguida, procedeu-se à determinação da relação caulinita/ (caulinita+gibbsita), com os dados de ATG, aqui chamados de $\mathrm{RCGb}_{\mathrm{ATG}}$.

Além do transecto, realizou-se o fatiamento da imagem $\mathrm{RCGb}_{\text {scale }}$ para os intervalos estabelecidos $0,25-0,50,0,50-0,75$ e $0,75-1,00$, com o objetivo de agrupar diferentes classes de solos com relação ao valor Ki.
Por fim, testou-se a normalidade dos dados em todos os processamentos, por meio do teste de ShapiroWilk. Testou-se a similaridade estatística dos dados de RCGb, obtidos pelos processamentos dos dados Aster, com os obtidos por meio de ATG. Para tal, adotou-se o teste estatístico não paramétrico de Mann-Whitney (teste U), e para os que ajustaram à normalidade, aplicou-se o teste $\mathrm{t}$ de Student, ambos por meio do programa Bioestat 5.0. O teste $\mathrm{U}$ é considerado, segundo a literatura específica, como uma versão do teste $\mathrm{t}$ de Student para avaliar a heterogeneidade de duas amostras ordinais, aleatórias e independentes, que não obedecem aos parâmetros da curva normal.

\section{Resultados e Discussão}

Para ordenar os procedimentos e, portanto, identificar a imagem-produto e também os procedimentos mais adequados para a investigação da relação mineralógica caulinita/(caulinita+gibbsita), estabeleceu-se a ordem de relevância entre as análises realizadas, tendo-se feito primeiro a comparação entre o comportamento dos transectos $R C G b_{\text {scale }}$ e $R C G b_{\text {ATG }}$ e suas respectivas linhas de tendências, seguida da identificação da espacialização da relação (caulinita/(caulinita + gibbsita) nas imagens, por meio do fatiamento dos valores de RCGb; e, finalmente, a análise estatística entre os valores dos transectos $\mathrm{RCGb}_{\text {scale }}$ e $\mathrm{RCGb}_{\mathrm{ATG}}$.

A comparação entre o comportamento dos transectos $\mathrm{RCGb}_{\text {scale }}$ e $\mathrm{RCGb}_{\mathrm{ATG}}$ foi considerada como a mais relevante, por fornecer os valores nominais da relação mineralógica investigada, em cada ponto dos transectos da imagem $\mathrm{RCGb}_{\text {scale, e compará-los com os valores }}$ da relação obtida por meio da ATG nas amostras de campo, realizada por Baptista (2001).

Do total dos procedimentos realizados, $70 \%$ apresentaram o comportamento da linha de tendência dos transectos $\mathrm{RCGb}_{\text {scale }}$ similares ao do transecto do $\mathrm{RCGb}_{\mathrm{ATG}}$. O transecto $\mathrm{RCGb}_{\text {scale }}$ que apresentou o comportamento com maior similaridade ao transecto $\mathrm{RCGb}_{\mathrm{ATG}}$ foi o obtido a partir dos dados de reflectância L1B, com a aplicação dos módulos Flaash e com correção "crosstalk". Esse pré-processamento também foi o utilizado por Vicente \& Souza Filho (2011), para a identificação de minerais de solos por meio de dados Aster. Esses autores utilizaram outro programa para a correção dos efeitos atmosféricos, porém, com base no mesmo modelo de transferência radiativa utilizado 
pelo Flaash, o MODTRAN 4. Caccetta et al. (2013) também optaram pelo produto L1B, com a correção “crosstalk", para a identificação mineralógica em escala continental na Austrália, porém, trabalharam com a reflectância TOA.

Os transectos $\mathrm{RCGb}_{\text {scale }}$ e $\mathrm{RCGb}_{\mathrm{ATG}}$ apresentaram tendências similares, calculadas a partir do ajuste de polinômios de quarto grau e plotadas nas linhas de tendência. Observou-se, também que, mesmo que o $\mathrm{RCGb}_{\text {scale }}$ tenha apresentado alguns valores super e subestimados, em relação aos respectivos valores do RCGb obtidos a partir da ATG, as escalas entre os dois grupos mostraram-se significativamente próximas $(\alpha=0,05)$, o que também foi verificado por Lagacherie et al. (2008), quando estudaram a identificação de conteúdo de argila e de carbonato de cálcio no solo, em diversas escalas, da bancada até sistemas aerotransportados

Essas diferenças decorrentes entre a escala de campo e a da imagem, observadas no presente estudo, são relativamente comuns, como se pode verificar no trabalho de Genú \& Demattê (2012), em que comparam diversos atributos de solos por meio de sensores terrestres e orbitais. Schmid et al. (2008) encontraram também valores super e subestimados, quando compararam espectros de solos obtidos tanto em laboratório como por meio dos dados Aster, e notaram que, apesar de os dados apresentarem valores de albedo diferenciados entre os tipos de espectros, a tendência é verificada nos dois conjuntos de dados. Nota-se nos espectros apresentados por esses autores o efeito do "crosstalk", embora eles tenham utilizado dados corrigidos para esse efeito.

No presente estudo, deve-se salientar que todos os pontos amostrados em campo apresentaram magnetita, mineral opaco, em sua composição, o que tende a aplainar os espectros de reflectância (Baptista et al., 2011). Porém, para as áreas em que os solos estão inseridos em contextos máficos e ultramáficos, que apresentam incrementos nos conteúdos de magnetita, o Aster tem mostrado eficiência na espacialização das relações mineralógicas. Isso pode ser visto também no trabalho de Guha et al. (2015), que investigaram índices espectrais e fizeram análise de componentes principais aplicados a dados L1B, corrigidos para os efeitos atmosféricos com o Flaash, no Complexo Máfico-Ultramáfico inserido no Cráton Dharwar, Índia. e obtiveram bons resultados, mesmo com a presença dos minerais opacos.

Uma observação interessante, extraída da análise dos procedimentos realizados, foi a de que o transecto $\mathrm{RCGb}_{\text {scale, }}$, que apresentou o segundo melhor resultado, foi obtido a partir de dados L1B, com radiância registrada no sensor sem a aplicação das correções, que continha apenas a normalização dos valores por remoção do contínuo espectral.

O procedimento de remoção do contínuo espectral também é utilizado por Lagacherie et al. (2008), como forma de normalização para a comparação de espectros obtidos em diversas plataformas. Porém, a utilização de dados de radiância, normalmente, não é um procedimento usual na literatura, pois a maioria dos trabalhos visa a identificação de relações mineralógicas, por meio de feições de absorção em espectros de reflectância. Conforme destacado por Baptista et al. (2011), as feições se originam nos dados de radiância. E isso pode ser verificado no trabalho de El-Magd et al. (2015), que utilizaram dados de radiância do Aster, com correção apenas do efeito "crosstalk", para o mapeamento de mineralizações de ouro no Deserto Central Oriental do Egito. Ou seja, no que se refere à análise do comportamento dos perfis espectrais, verificou-se que os efeitos atmosféricos e do "crosstalk" não interferem na identificação da relação mineralógica. Isso já havia sido verificado em dados Aster, obtidos da mesma forma e com uso dos mesmos processamentos, em solos derivados de metassedimentos do grupo Paranoá, em São João D'Aliança, GO (Vivaldi et al., 2013).

Os $30 \%$ dos resultados restantes, referentes aos processamentos com dados L1A, apresentaram a linha de tendência dos transectos com comportamento aparentemente aplainado ou diferente, quando comparados na mesma escala de valores, o que impossibilitou ou prejudicou a comparação entre o comportamento dos transectos.

O processamento L1A convertido para reflectância de superfície (Flaash) foi o que forneceu os resultados menos satisfatórios. Os dados brutos L1A são utilizados nos algoritmos dos produtos sob demanda, ou para a construção de modelos digitais de terreno (MDT), em razão da possibilidade de aquisição de dados estereoscópicos, sem grandes alterações da imagem original (San \& Süzen, 2005). Mas os estudos disponíveis na literatura, normalmente, baseiam-se 
em dados L1B convertidos para reflectância, tanto de superfície, como TOA (Giglio et al., 2008). A Figura 3 apresenta o melhor e o pior resultado.

A espacialização da relação (caulinita/(caulinita+ gibbsita), por meio do fatiamento da imagem dos valores de RCGb, proporciona a espacialização dos solos mais cauliníticos ou gibbsíticos. Porém, é necessário que exista previamente uma noção da espacialização da relação mineralógica o que, neste caso, contava com o trabalho prévio de Baptista et al. (2011), por meio de dados Aviris, para a mesma mancha de solos aqui investigados. As classes adotadas foram: vermelho $(0,25-0,50)$; verde $(0,51-0,75)$; e azul $(0,76-1,00)$.

A espacialização da relação mineralógica foi observada, inicialmente, nas áreas indicadas pela cor vermelha, associada a áreas de porções mais intemperizadas, com o valor médio $\mathrm{RCGb}_{\text {scale }}$ de 0,44 para o ponto 1 do transecto. Nessa área, Baptista (2001) encontrou um Latossolo Vermelho distroférrico, predominantemente gibbsítico, com valor $\mathrm{Ki}=0,39$. Seguindo o transecto, os valores mudam para os de solos cauliníticos, com valor Ki médio de 0,6, representado, na Figura 4, pelos pixels na cor verde. Finalmente, o transecto chega ao Argissolo Vermelho, com maior valor $\mathrm{Ki}$, ao redor de 0,8 , caracterizado pelos pixels de cor azul na área com os maiores teores de caulinita. Reatto et al. (2008) consideram que a variabilidade mineralógica regional, no Planalto Central, está associada à idade da superfície, pois, nas mais antigas áreas ocorre mais remoção de sílica $\left(\mathrm{SiO}_{2}\right)$ e predominam ambientes mais gibbsíticos do que cauliníticos. Porém, esses mesmos autores salientam que, localmente, as condições hidráulicas ao longo da topossequência são o que determinam a variabilidade mineralógica. O Latossolo Vermelho distroférrico, no presente estudo, situa-se em uma depressão côncava que expressa maiores conteúdos gibbsíticos e, na medida que caminha o transecto em direção ao Argissolo, as áreas apresentam-se mais convexas e, provavelmente, menos intemperizadas, formadas por leques aluviais transportados de montante, que expressam maiores conteúdos de caulinita (Baptista et al., 2011). Essas mesmas relações entre a topografia da área e a mineralogia foram encontradas por Galvão et al. (2008), por meio da integração de dados hiperespectrais do Aviris, com o MDT gerado pelo par estereoscópico do Aster, para a região de Campo Verde, MT.

Das 10 imagens $\mathrm{RCGb}_{\text {scale }}$ fatiadas, $80 \%$ apresentaram resultados que proporcionaram a visualização do fluxo da espacialização dos minerais, e a imagem obtida a partir dos dados L1B (radiância TOA) foi a que apresentou o resultado mais coerente com a variabilidade mineralógica apresentada por Baptista et al. (2011), obtida por meio de dados Aviris. $\mathrm{O}$ restante não permitiu a espacialização da relação mineralógica existente na área. A Figura 4 apresenta a imagem $\mathrm{RCGb}_{\text {scale }}$ fatiada, referente ao resultado
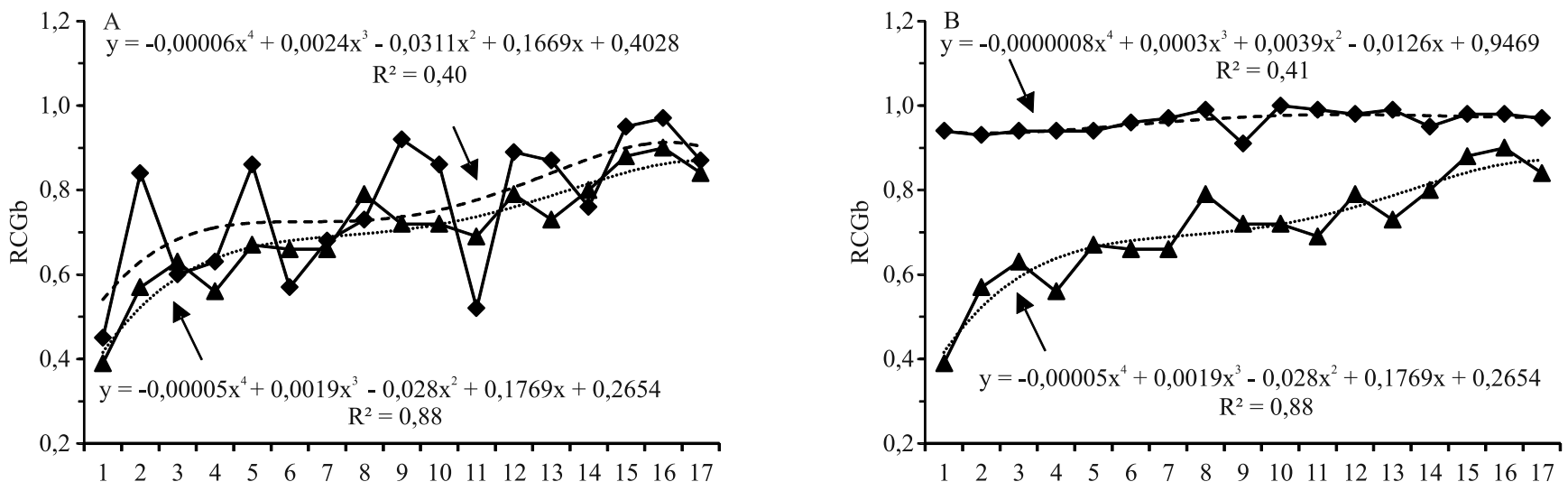

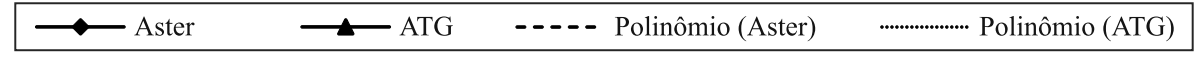

Figura 3. Melhor resultado em transectos de RCGb, obtidos por meio de ATG e dos dados L1B convertidos para reflectância de superfície com correção atmosférica e de "crosstalk" (A); e pior resultado em transectos RCGb ATG $_{\text {e RCGb }}$ scale $_{\text {dos dados L1A }}$ convertidos para reflectância de superfície, por meio da correção atmosférica com Flaash e sem correção de "crosstalk" (B). 
que mais se aproximou da variabilidade mineralógica da área, e a que não ressaltou essa variabilidade. A ideia de se comparar a espacialização da relação mineralógica, obtida com dados Aster, com a obtida por meio do Aviris, também foi realizada por Mars \& Rowan (2010) na região de Cuprite, NV. Esses autores utilizaram dados L1B convertidos para reflectância de superfície, com correção de "crosstalk" e com o produto sob demanda AST07XT.

Quanto se fez a análise estatística entre os valores dos transectos $\mathrm{RCGb}_{\text {scale }}$ e $\mathrm{RCGb}_{\mathrm{ATG}}$, a verificação da normalidade dos transectos foi realizada por meio do teste de Shapiro-Wilk, que mostrou que $70 \%$ dos dados não apresentaram distribuição normal. Nesse sentido, optou-se pelo teste de hipótese de similaridade, entre os valores obtidos nos processamentos com os dados de RCGb e os obtidos por ATG, tendo-se, então utilizado teste U não paramétrico de Mann-Whitney. Considerou-se, para a aplicação do teste, o valor do $\mathrm{U}_{\mathrm{tab}}=87$, os valores críticos $\alpha=0,05$, tamanho de amostra $=17 \mathrm{e}$, como condicionantes, se $\mathrm{U}_{\text {calc }} \geq \mathrm{U}_{\text {tab }}$, então $\mathrm{RCGb}_{\text {scale }}$ e $\mathrm{RCGb}_{\mathrm{ATG}}$ não apresentaram similaridade estatística; se $\mathrm{U}_{\text {calc }}<\mathrm{U}_{\text {tab}}$, então $\mathrm{RCGb}_{\text {scale }}$ e $\mathrm{RCGb}_{\mathrm{ATG}}$ apresentaram similaridade estatística. Para os 30\% dos dados restantes que apresentaram distribuição normal, procedeu-se ao teste $t$ de Student.

Entre os sete processamentos testados com Mann Whitney, seis rejeitaram a hipótese nula de similaridade estatística entre os valores dos transectos do $\mathrm{RCGb}_{\text {scale }}$ e do $\mathrm{RCGb}_{\mathrm{ATG}}$. Para os três processamentos que apresentaram distribuição normal e foram submetidos ao teste $\mathrm{t}$ de Student, dois aceitaram a hipótese nula, ou seja, apresentaram similaridade estatística entre os transectos de imagem e de campo. Isso significa que, de acordo com o teste estatístico aplicado, a combinação - entre os módulos de pré-tratamentos dos dados, os módulos aplicados para cada um dos procedimentos e a aplicação do índice $\mathrm{RCGb}_{\text {scale }}-$, pode ter influenciado o distanciamento dos valores dos transectos do $\mathrm{RCGb}_{\text {scale }}$ e do $\mathrm{RCGb}_{\mathrm{ATG}}$ e, consequentemente, o grau de correlação, o que torna as amostras distintas entre si e rejeita a hipótese de igualdade das medianas. O mesmo fato foi verificado por Vivaldi et al. (2013), quando avaliaram os solos no contexto metassedimentar de São João D'Aliança, GO.

Para o ordenamento das imagens Aster-SWIR, atribuíram-se pesos de acordo como grau de importância das três análises, definidas anteriormente, que foram: análise 1 , comparação entre o comportamento dos transectos $\mathrm{RCGb}_{\text {scale }}$ e $\mathrm{RCGb}_{\text {ATG}}$, peso 3 ; análise 2, espacialização de RCGb por meio de fatiamento, peso 2; e análise 3 , análise estatística entre os valores dos transectos $\mathrm{RCGb}_{\text {scale }}$ e RCGb $\mathrm{RTG}_{\mathrm{ATG}}$, peso 1 .

Conclui-se, portanto, que a imagem $\mathrm{RCGb}_{\text {scale }}$ obtida com dados L1B de reflectância TOA ("flat field"), sem correção atmosférica e de "crosstalk", foi ranqueada como a mais adequada para a investigação da relação mineralógica nessa mancha de solos de Niquelândia, rica em minerais opacos. $\mathrm{E}$ o menos adequado foi o processamento com dados brutos L1A, corrigidos pelo Flaash e sem correção de "crosstalk". Há coerência entre os resultados observados no presente estudo,
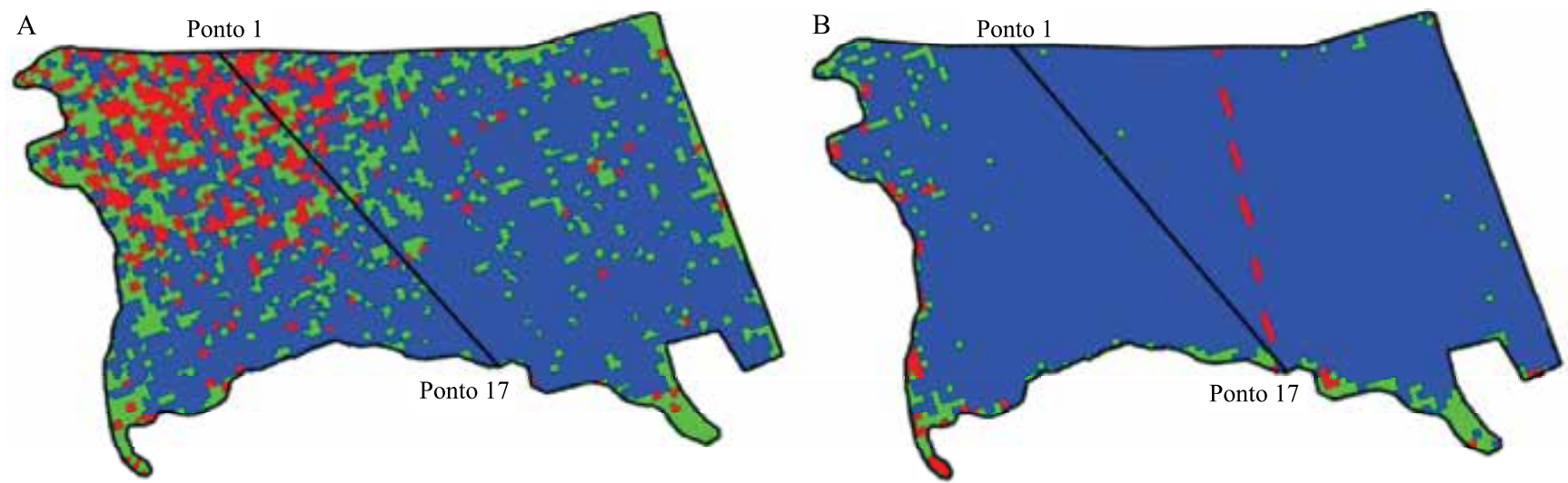

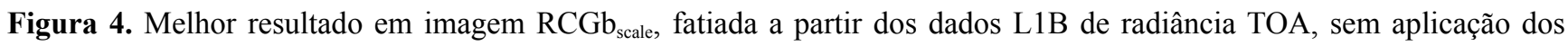

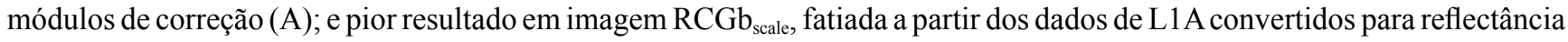
de superfície, por meio da correção atmosférica com Flaash e sem correção de "crosstalk" (B). 
pois, como salientado anteriormente, é comum a utilização de dados de radiância no nível do sensor (TOA), na maioria dos trabalhos investigados. Assim, a literatura é ainda carente de estudos com dados L1A, bem como de investigação da potencialidade dos diversos produtos Aster, para a espacialização da relação mineralógica investigada no presente trabalho.

Vivaldi et al. (2013) encontraram na imagem reflectância L1B, com correção "crosstalk" (CRSTK30) e com correção atmosférica (Quac), bem como na imagem AST_07, os melhores resultados. A pior imagem foi a obtida com dados L1B convertidos para reflectância de superfície (Flaash), com correção "crosstalk" para os dados Aster, do Município de São João D'Aliança. Essa divergência, principalmente quando se compara o pior resultado, decorre provavelmente da aplicação de um modelo complexo de correção atmosférica, o que não foi observado no presente estudo.

\section{Conclusões}

1. Apesar do efeito "crosstalk", é possível identificar a relação mineralógica RCGb: caulinita/ (caulinita+gibbsita) a partir de dados Aster, em uma mancha de solos expostos derivados do Complexo de Niquelândia, constituído principalmente por gabronoritos, noritos e piroxenitos, rico em minerais opacos, como a magnetita.

2. O resultado ranqueado como o mais adequado para a investigação da relação mineralógica foi $\mathrm{o}$ obtido a partir de dados L1B de reflectância TOA ("flat field"), sem correção atmosférica e de "crosstalk".

3. Novos estudos sobre a potencialidade dos dados Aster, para a compreensão de coberturas pedológicas, devem ser incentivados.

\section{Referências}

ABRAMS, M.; HOOK, S.; RAMACHANDRAN, B. Aster user handbook: version 2. 2007. Disponível em: <http://Asterweb. jpl.nasa.gov/content/03_data/04_Documents/Aster_user_guide_ v2.pdf $>$. Acesso em: 17 ago 2015.

BALDRIDGE, A.M.; HOOK, S.J.; GROVE, C.I.; RIVERA, G.G. The Aster spectral library version 2.0. Remote Sensing of Environment, v.113, p.711-715, 2009. DOI: $10.1016 / \mathrm{j}$. rse.2008.11.007.

BAPTISTA, G.M. de M. Mapeamento e quantificação da relação mineralógica caulinita/(caulinita+gibbsita) de solos tropicais, por meio dos dados do sensor hiperespectral Aviris
(JPL/NASA). 2001. 139p. Tese (Doutorado) - Universidade de Brasília, Brasília.

BAPTISTA, G.M.M.; CORREA, R.S.; SANTOS, P.F. dos; MADEIRA NETTO, J.S.; MENESES, P.R. Use of imaging spectroscopy for mapping and quantifying the weathering degree of tropical soils in central Brazil. Applied and Environmental Soil Science.v.2011, article ID 641328, p. 1-7, 2011.

BAPTISTA, G.M. de M.; CORREA, R.S.; TORRES, M.G.; BIAS, E. de S.; RESENDE, M.G.; RIBEIRO, R.J. da C.; SILVA, D.J. da. Identificação de relação mineral caulinita/gibbsita em solos tropicais, por meio dos dados SWIR do sensor Aster. In: SIMPÓSIO BRASILEIRO DE SENSORIAMENTO REMOTO, 13., 2007, Florianópolis, SC. Anais. São José dos Campos: INPE, 2007. p.7043-7050.

BERNSTEIN, L.S.; JIN, X.; GREGOR, B.; ADLER-GOLDEN, S.M. Quick atmospheric correction code: algorithm description and recent upgrades. Optical Engineering, v.51, p.111719-111730, 2012. DOI: 10.1117/1.OE.51.11.111719.

CACCETTA, M.; COLLINGS, S.; CUDAHY, T. A calibration methodology for continental scale mapping using Aster imagery. Remote Sensing of Environment, v.139, p.306-317, 2013. DOI: 10.1016/j.rse.2013.08.011

CLARK, R.N.; GALLAGHER, A. J.; AND SWAYZE, G.A. 1990: Material absorption band depth mapping of imaging spectrometer data using the complete band shape least-squares algorithm simultaneously fit to multiple spectral features from multiple materials. In: AIRBORNE VISIBLE/INFRARED IMAGING SPECTROMETER (Aviris), 3., Pasadena, 1990. Proceedings. Pasadena: JPL Publication 90-54, 1990. p.176-186.

EL-MAGD, I.A.; MOHY, H.; BASTA, F. Application of remote sensing for gold exploration in the Fawakhir area, Central EAstern Desert of Egypt. Arabian Journal of Geosciences, v.8, p.35233536, 2015. DOI: 10.1007/s12517-014-1429-4.

FERREIRA FILHO, C.F.; PIMENTEL, M.M.; ARAUJO, S.M. de; LAUX, J.H. Layered intrusions and volcanic sequences in central Brazil: geological and geochronological constraints for Mesoproterozoic $(1.25 \mathrm{Ga})$ and Neoproterozoic $(0.79 \mathrm{Ga})$ igneous associations. Precambrian Research, v.183, p.617-634, 2010. DOI: 10.1016/j.precamres.2010.06.008.

GALVÃO, L.S.; ALMEIDA-FILHO, R.; VITORELLO, Í. Spectral discrimination of hydrothermally altered materials using Aster short-wave infrared bands: evaluation in a tropical savannah environment. International Journal of Applied Earth Observation and Geoinformation, v.7, p.107-114, 2005. DOI: 10.1016/j.jag.2004.12.003.

GALVÃO, L.S.; FORMAGGIO, A.R.; COUTO, E.G.; ROBERTS, D.A. Relationships between the mineralogical and chemical composition of tropical soils and topography from hyperspectral remote sensing data. ISPRS Journal of Photogrammetry and Remote Sensing, v.63, p.259-271, 2008. DOI: 10.1016/j. isprsjprs.2007.09.006.

GENÚ, A.M.; DEMATTÊ J.A.M. Espectrorradiometria de solos e comparação com sensores orbitais. Bragantia, v.71, p.82-89, 2012. DOI: $10.1590 / \mathrm{S} 0006-87052012005000005$. 
GIGLIO, L.; CSISZAR, I.; RESTÁS, Á.; MORISETTE, J.T.; SCHROEDER, W.; MORTON, D.; JUSTICE, C.O. Active fire detection and characterization with the advanced spaceborne thermal emission and reflection radiometer (Aster). Remote Sensing of Environment, v.112, p.3055-3063, 2008. DOI: 10.1016/j.rse.2008.03.003.

GUHA, A.; GHOSH, B.; KUMAR, K.V.; CHAUDHURY, S. Implementation of reflection spectroscopy based new Aster indices and principal components to delineate chromitite and associated ultramafic-mafic complex in parts of Dharwar Craton, India. Advances in Space Research, v.56, p.1453-1468, 2015. DOI: 10.1016/j.asr.2015.06.043.

IWASAKI, A.; TONOOKA, H. Validation of a crosstalk correction algorithm for Aster/SWIR. IEEE Transactions Geoscience and Remote Sensing, v.43, p.2747-2751, 2005. DOI: 10.1109/ TGRS.2005.855066.

LAGACHERIE, P.; BARET, F.; FERET, J.-B.; MADEIRA NETTO, J.S.; ROBBEZ-MASSON, J.M. Estimation of soil clay and calcium carbonate using laboratory, field and airborne hyperspectral measurements. Remote Sensing of Environment, v.112, p.825-835, 2008. DOI: 10.1016/j.rse.2007.06.014.

MADEIRA NETTO, J. da S. Étude quantitative des relations constituants minéralogiques - réflectance diffuse des Latosols brésiliens: application à l'utilisation pédologique des données satellitaires TM (région de Brasilia). Paris: Orstom, 1993. 236p.

MARS, J.C.; ROWAN, L.C. Spectral assessment of new Aster SWIR surface reflectance data products for spectroscopic mapping of rocks and minerals. Remote Sensing of Environment, v.114, p.2011-2025, 2010. DOI: 10.1016/j.rse.2010.04.008.

PERKINS, T.; ADLER-GOLDEN, S.M.; MATTHEW, M.W.; BERK, A.; BERNSTEIN, L. S.; LEE, J.; FOX, M. Speed and accuracy improvements in Flaash atmospheric correction of hyperspectral imagery. Optical Engineering, v.51, p.111707111714, 2012. DOI: 10.1117/1.oe.51.11.111707.

PLANTE, A.F.; FERNÁNDEZ, J.M.; LEIFELD, J. Application of thermal analysis techniques in soil science. Geoderma, v.153, p.110, 2009. DOI: 10.1016/j.geoderma.2009.08.016.

REATTO, A.; BRUAND, A.; MARTINS, E. de S.; MULLER, F.; SILVA, E.M. da; CARVALHO JÚNIOR, O.A. de; BROSSARD, $\mathrm{M}$. Variation of the kaolinite and gibbsite content at regional and local scale in Latosols of the Brazilian Central Plateau. Comptes Rendus Geoscience, v.340, p.741-748, 2008. DOI: 10.1016/j. crte.2008.07.006.

SAN, B.T.; SÜZEN, M.L. Digital elevation model (DEM) generation and accuracy assessment from Aster stereo data for environmental research. International Journal of Remote Sensing, v.26, p.5013-5027, 2005.

SCHMID, T.; KOCH, M.; DIBLASI, M.; HAGOS, M. Spatial and spectral analysis of soil surface properties for an archaeological area in Aksum, Ethiopia, applying high and medium resolution data. Catena, v.75, p.93-101, 2008. DOI: 10.1016/j.catena.2008.04.008.

VICENTE, L.E.; SOUZA FILHO, C.R. de. Identification of mineral components in tropical soils using reflectance spectroscopy and advanced spaceborne thermal emission and reflection radiometer (Aster) data. Remote Sensing of Environment, v.115, p.18241836, 2011. DOI: 10.1016/j.rse.2011.02.023.

VIVALDI, D.D.; BAPTISTA, G.M. de M.; MENESES, P.R. Avaliação dos processamentos de crosstalk e de correção atmosférica em dados Aster para identificação da relação mineralógica caulinita/(caulinita + gibbsita) em solos no Município de São João d'Aliança (GO). Brazilian Journal of Geology, v.43, p.571-582, 2013. DOI: 10.5327/Z231748892013000300011.

Recebido em 26 de agosto de 2015 e aprovado em 10 de março de 2016 